\title{
Single Mode Broad Area PT-Symmetric Microring Lasers
}

\author{
W. E. Hayenga, M.-A. Miri, H. Hodaei, A. U. Hassan, M. Heinrich, D. N. Christodoulides, M. Khajavikhan* \\ CREOL, The College of Optics and Photonics, University of Central Florida, Orlando, Florida 32816-2700, USA \\ *mercedeh@,creol.ucf.edu
}

\begin{abstract}
Single-transverse mode lasing is demonstrated in a broad-area PT-symmetric semiconductor microring arrangement. The proposed scheme is versatile, robust to fabrication errors, and allows for high brightness operation while maintaining spectral purity.

OCIS codes: (140.3570) Lasers, single-mode; (140.3948) Microcavity devices; (140.3410) Laser resonators
\end{abstract}

Broad-area lasers provide high output powers while managing the thermal load and reducing the impact of optical nonlinearities $[1,2]$. Unfortunately, enlarging the cross section of a waveguide inevitably introduces higher order transverse modes and leads to undesired filamentation [3], thus compromising the spectral and spatial fidelity of the laser and limiting the brightness of the output beam. These limitations exist on all scales, and may even be exacerbated in chip-scale semiconductor lasers, where the large gain bandwidths afforded by the active media already pose a challenge in promoting single-mode operation. Tapering along the direction of propagation and/or engineering the refractive index in the cross section have been extensively pursued as possible techniques to enforce single mode operation in such broad area semiconductor arrangements. Despite their success in suppression of higher order modes, the application of these techniques is quite limited because of their inherent sensitivity to perturbations. Lately, the selective breaking of parity-time (PT) symmetry has been proposed as a viable strategy for obtaining single transverse mode operation [4]. Along these lines, by pairing an active resonator/waveguide with a lossy but otherwise identical partner, it is possible to enforce single-spatial-mode performance, even in the presence of strong mode competition in broad area laser/amplifier configurations [4].

Here we experimentally show that parity-time symmetry can be utilized in promoting the fundamental transverse mode in broad-area microring lasers. In this case, the virtual threshold imposed by PT symmetric exceptional points introduces an additional degree of freedom in terms of the attainable gain enhancement. In this regard, higher order spatial modes exhibit stronger coupling coefficients because they are less confined, and as a result, in a PT arrangement the fundamental mode is the first in line to break its symmetry as the gain increases, thus experiencing a net amplification. On the other hand, for this same gain level, the rest of the modes exhibit an unbroken symmetry and therefore remain entirely neutral. Indeed, following our approach, one can globally enforce single-mode behavior both in the spatial and spectral domain without compromising the efficiency of the system [5].
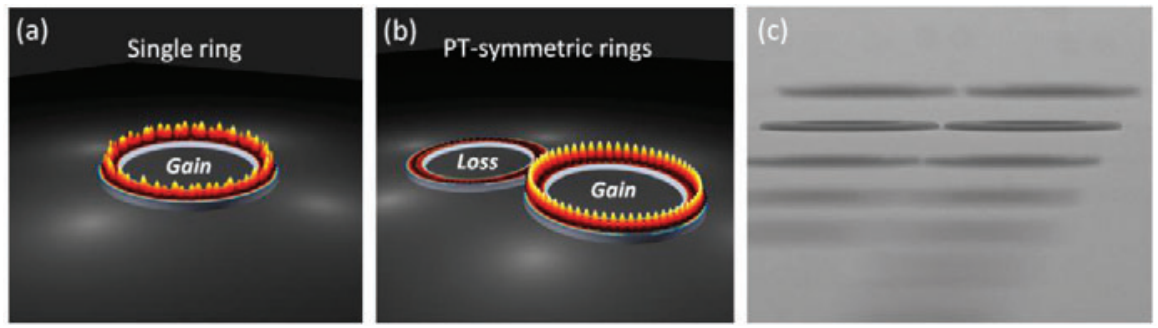

Fig. 1. (a) In a single microring resonator, multiple transverse and longitudinal modes can lase simultaneously, (b) in the PT-symmetric arrangement, on the other hand, only one longitudinal mode with the lowest order transverse distribution enjoys lasing, and (c) an SEM image of a PT-symmetric double ring laser arrangement.

The schematics of Figs. 1(a) and (b) depict the envisioned transition from a multimode behavior in a broad area microring laser to single mode operation in a double ring configuration as enabled by preferential PT symmetry breaking. Our experiments were conducted in high-contrast active ring resonators based on InGaAsP (Indium-Gallium-Arsenide-Phosphide) multiple quantum wells embedded in $\mathrm{SiO}_{2}$ (silicon dioxide) as shown in Fig. 1(c). Based on our measurements, we estimate the quality factor of the fabricated microrings to be on the order of 120,000. The gain bandwidth of the active medium spans the spectral region between 1260 and $1590 \mathrm{~nm}$. Whereas the quantum wells are present in all wave-guiding sections, gain and loss is provided by selectively pumping the respective rings (pump wavelength: $1064 \mathrm{~nm}$ ). Accordingly, the effective pump powers are proportional to the geometric overlap between the active medium and the pump profile. The intensity distribution and the spectrum of the light oscillating in the microrings were then monitored by means of an infrared camera and a grating spectrometer, respectively [5]. Rings with radii of $R=6 \mu \mathrm{m}$ and waveguide dimensions of $0.21 \mu \mathrm{m} \times 1.5 \mu \mathrm{m}$ were chosen for their comparatively large free spectral range $\left(\Delta \lambda_{\mathrm{FSR}} \sim 20 \mathrm{~nm}\right)$ and readily discernible transverse mode sets. 
The geometries used in our experiments corresponding to a single and a double PT-symmetric configuration are shown in Figs. 2(a) and (b). Fig. 2(c) depicts the spectrum obtained from a single microring laser that can support up to four transverse modes. In this case, due to differences in confinement, only the $\mathrm{TE}_{0}$ and $\mathrm{TE}_{1}$ sets are lasing at moderate pump power levels. As expected, no mode selectivity between these two sets of modes is observed as the pump power is decreased (Fig. 2(d)). On the other hand, Fig. 2(e) depicts the lasing spectrum of a PT-symmetric double-ring arrangement when the active ring is supplied with the same pump power as in Fig. 2(c). Under these conditions, the $\mathrm{TE}_{1}$ subset falls below the PT breaking threshold, and therefore experiences zero net gain. The corresponding resonances are suppressed down to the noise level with over $30 \mathrm{~dB}$ fidelity. Finally, by decreasing the pump power to the level of Fig. 2(f), global single mode operation (spectrally and spatially) can be achieved in this paired microring system, as only one single longitudinal resonance of the fundamental $\mathrm{TE}_{0}$ mode experiences sufficient gain to induce PT symmetry breaking. It should be emphasized that the PT single-mode gain systematically exceeds the maximum achievable gain discrimination in a single microring configuration.

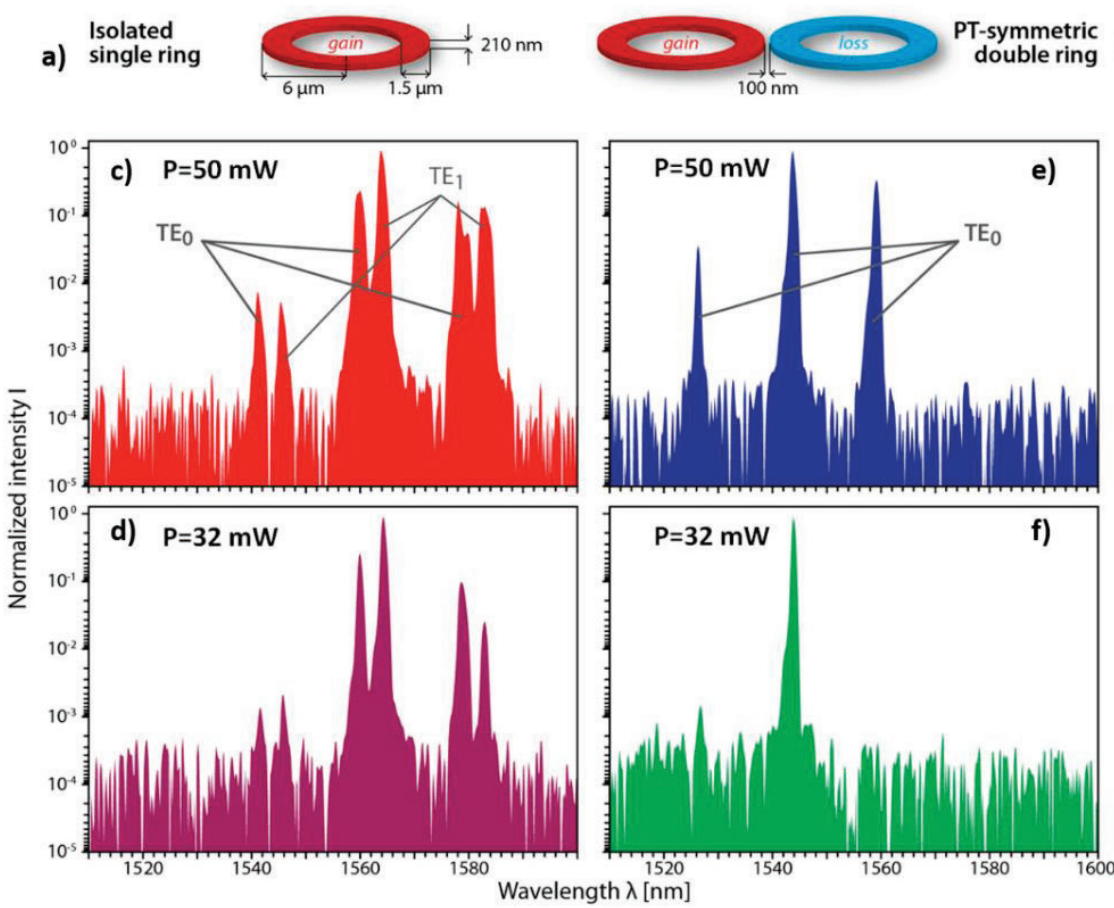

Fig. 2. Experimental results. (a) and (b) depict the geometries of a single ring and the double PT-symmetric configuration. (c) A single microring resonator lases in various $\mathrm{TE}_{0}$ and $\mathrm{TE}_{1}$ modes, (d) by decreasing the pump power no mode selectivity is observed, (e) in contrast, a PT-symmetric double ring arrangement reliably suppresses all the $\mathrm{TE}_{1}$ modes with a contrast exceeding $30 \mathrm{~dB}$, while the remaining $\mathrm{TE}_{0}$ set is unaffected, and (f) global single-mode operation with similar fidelity can be enforced by adjusting the pump power such that only one longitudinal resonance of the fundamental $\mathrm{TE}_{0}$ mode undergoes $\mathrm{PT}$ symmetry breaking.

In conclusion, in this work we have experimentally shown the spatial mode filtering properties of the parity-time symmetric structures for the first time. Moreover, we have demonstrated simultaneous single transverse and longitudinal mode operation in PT-symmetric broad area microring lasers. This was achieved by appropriately accompanying an active microring resonator with an identical but lossy counterpart which acts as a shape- and wavelength-selective filter for all undesired modes. Unlike other filtering schemes, the proposed method establishes mode selectivity through coupling to the lossy cavity without compromising the optical power extracted from the fundamental mode. Our approach is versatile, scalable and can be applied to a wide range of broad area laser systems. Finally, the PT-symmetric microring configurations considered here can be easily incorporated in existing semiconductor-based integrated photonic platforms.

\section{REFERENCES}

[1] D. F. Welch, “A brief history of high-power semiconductor lasers," J. Sel. Top. Quantum Electron. 6 1470-1477 (2000).

[2] J. J. Plant et. al. “1.5- $\mu \mathrm{m}$ InGaAsP-. InP slab-coupled optical waveguide lasers” IEEE Photon. Technol. Lett. 17, 735 (2005).

[3] D. Mehuys, M. Mittelstein, J. Salzman, and A. Yariv, "Saturable nonlinear dielectric waveguide with applications to broad-area semiconductor lasers" Opt. Lett. 12, 953 (1987).

[4] M.-A. Miri, P. LiKamWa and D. N. Christodoulides, "Large area single-mode parity-time-symmetric laser amplifiers" Opt. Lett. 37, 764 (2012).

[5] H. Hodaei, M.-A. Miri, M. Heinrich, D.N. Christodoulides, and M. Khajavikhan, “PT-symmetric microring lasers,” Science 346975 (2014). 